\title{
Numerical study of fluxon dynamics in a system of two-stacked Josephson junctions
}

\author{
Petraglia, Antonio; Ustinov, A. V.; Pedersen, Niels Falsig; Sakai, S.
}

Published in:

Journal of Applied Physics

Link to article, DOI:

10.1063/1.359547

Publication date:

1995

Document Version

Publisher's PDF, also known as Version of record

Link back to DTU Orbit

Citation (APA):

Petraglia, A., Ustinov, A. V., Pedersen, N. F., \& Sakai, S. (1995). Numerical study of fluxon dynamics in a system of two-stacked Josephson junctions. Journal of Applied Physics, 77(3), 1171-1177.

https://doi.org/10.1063/1.359547

\section{General rights}

Copyright and moral rights for the publications made accessible in the public portal are retained by the authors and/or other copyright owners and it is a condition of accessing publications that users recognise and abide by the legal requirements associated with these rights.

- Users may download and print one copy of any publication from the public portal for the purpose of private study or research.

- You may not further distribute the material or use it for any profit-making activity or commercial gain

- You may freely distribute the URL identifying the publication in the public portal

If you believe that this document breaches copyright please contact us providing details, and we will remove access to the work immediately and investigate your claim 


\title{
Numerical study of fluxon dynamics in a system of two-stacked Josephson junctions
}

\author{
A. Petraglia \\ Physics Department, Technical University of Denmark, DK-2800 Lyngby, Denmark \\ A. V. Ustinov \\ Institute of Thin Films and Ion Technology, Research Center (KFA), D-52425 Jülich, Germany \\ N. F. Pedersen \\ Physics Department, Technical University of Denmark, DK-2800 Lyngby, Denmark \\ S. Sakai \\ Electrotechnical Laboratory, 1-1-4 Umezono, Tsukuba-shi, Ibaraki 305, Japan
}

(Received 30 June 1994; accepted for publication 4 October 1994)

The dynamics of magnetic fluxons in a system of two vertically stacked long Josephson junctions is investigated numerically. The model is based on the approach by S. Sakai, P. Bodin, and N. F. Pedersen [J. Appl. Phys. 73, 2411 (1993)] and is described by two strongly cóbled sine-Gordon equations. In agreement with recent experimental data, we confirm numerically the effect of splitting of the fluxon travelling mode into two separated modes with different characteristic velocities. The simulated current-voltage characteristics indicate stable phase-locked flux-flow resonances of two junctions. These results support a possibility of application of the stacked long Josephson junctions as a system of coherent oscillators for millimeter and sub-millimeter wave bands. 1995 American Institute of Physics.

\section{INTRODUCTION}

Systems consisting of two or more stacked long Josephson junctions have recently been studied experimentally by several groups. ${ }^{1-5}$ Although such systems have mostly been realized as stacks of $\mathrm{Nb}-\mathrm{Al} / \mathrm{AlO}_{x}-\mathrm{Nb}$ tunnel junctions, there is also interest in such systems as a model of layered BSCCO high- $T_{c}$ superconductors. ${ }^{6}$ Thus there have already been published experiments clearly demonstrating that $\mathrm{BSCCO}$ high- $T_{c}$ superconductors may be considered as a stack of Josephson junctions. ${ }^{7}$

On the theoretical side several aspects of the stacked long Josephson junction problem has been studied. In Ref. 8 a theoretical foundation for describing the system-based on the Maxwell, London, and Josephson equations--were established, and a few predictions for likely experimental results were pointed out. More exotic and less experimentally feasible $n$-dimensional systems were studied theoretically and numerically in Ref. 9.

The general problem of two coupled long Josephson junctions has been first formulated by Mineev et al. ${ }^{10}$ and has been studied by several groups. ${ }^{11-14}$ In most of those cases it has been assumed that the inductive coupling between the junctions was horizontal ${ }^{15}$ (and small) as opposed vertical $^{1-5}$ (and possibly large) as in the case here. Although the precise nature of the coupling in the previous papers ${ }^{10-11}$ was not specified as in the case of the stacked junction model, ${ }^{8}$ many general features of the dynamics of coupled systems can be learned.

The purpose of the present work is to study numerically the variety of dynamical behaviors that can be expected in systems of two-stacked long Josephson junction systems. Previously the identified modes of dynamical behaviors are
- coherent (in-phase) motion of two fluxons ${ }^{8,14}$ (one in each junction),

- symmetric motion (antiphase) of two fluxons ${ }^{8,14}$ (one in each junction),

- flux flow steps with Fiske resonances (experimental observation $^{2}$ ),

- coherent motion of fluxon arrays in two junctions in a flux flow mode (experimental observation ${ }^{3}$ ).

In our numerical investigations we change the relevant parameters for the two junction system, including the boundary conditions and the biasing conditions for the two junctions. By doing this we are able to confirm and elaborate on the modes listed above and to identify new types of dynamical behavior. In agreement with experiment, ${ }^{2}$ the simulations indicate a splitting of the dispersion relation in two branches, each of them corresponding to fluxons moving with different Swihart velocittes. Ir addition, we were able to simulate the current-voltage characteristics of the stack which were previously observed experimentally and understood to be the phase-locked flux-flow'resonances of the two junctions.

Simulation results presented here support a possibility of using stacked long Josephson junctions as local oscillators for millimeter and sub-millimeter wave cryoelectronic circuits. Stacked junctions have several advantages with respect to planar junction arrays, in particular due to the stronger coupling between individual junctions, highly integrated design, and rather simple fabrication requirements. Vertical stacking of long junctions is expected to increase the impedance of the whole structure which simplifies the impedance matching requirements for the external load. First experiments on phase locking in stacks ${ }^{3}$ showed very wide tuneable frequency range of coherent operation of stacked junctions. 
The paper is organized in the following way. Section II discusses briefly the fundamentals of the mode splitting of the Swihart velocity that is essential to the understanding of the dynamic behavior of the two junction stack. Section III contain numerical simulations for the simple system with annular geometry. In the subsections here we consider different numbers of fluxons in the two junctions, different bias conditions for the two junctions and the possibility of fluxon-, antifluxon coherent motion. In Sec. IV we consider two stacked junctions in the overlap geometry and here we study the interaction of fluxons with small amplitude electromagnetic waves (Fiske modes) and the magnetic field dependence. We also try different bias conditions for the two junctions. Finally, we study the influence of changes of the coupling parameter, which for the stacked junctions is not restricted to small values. Section V contains the conclusion and suggestions for new experiments to explore the dynamics of the stacked two Josephson junction system.

\section{THEORY}

The coupled sine-Gordon equations that will be used in the simulations describe two coupled long Josephson junctions $\mathrm{A}$ and $\mathrm{B}$ as derived in Ref. 8:

$$
\left(\begin{array}{c}
\varphi_{x x}^{\mathrm{A}} \\
\varphi_{x x}^{\mathrm{B}}
\end{array}\right)=\left(\begin{array}{cc}
1 & S \\
S & 1
\end{array}\right)\left(\begin{array}{c}
\varphi_{t t}^{\mathrm{A}}+\alpha \varphi_{t}^{\mathrm{A}}+\sin \varphi^{\mathrm{A}}-\gamma_{\mathrm{A}} \\
\varphi_{t t}^{\mathrm{B}}+\alpha \varphi_{t}^{\mathrm{B}}+\sin \varphi^{\mathrm{B}}-\gamma_{\mathrm{B}}
\end{array}\right) .
$$

Here the coupling parameter is defined as

$$
S=\frac{s}{d^{\prime}}=-\frac{\lambda_{L}}{\sinh \left(t / \lambda_{L}\right)\left(d+\lambda_{L} \operatorname{coth}\left(t / \lambda_{L}\right)+\lambda_{L} \operatorname{coth}\left(t_{e} / \lambda_{L}\right)\right)} .
$$

We have assumed that junctions $A$ and $B$ are identical (symmetric stack). In the equation the barrier thickness is $d$, the middle electrode thickness is $t$, and the top and bottom electrode thickness is $t_{e}$, while the London penetration depth is called $\lambda_{L}$. The spatial coordinate $x$ is normalized to the Josephson penetration depth $\lambda_{J}=\left[\Phi_{0} /\left(2 \pi \mu_{0} d^{\prime} j_{c}\right)\right]^{1 / 2}$, where $\Phi_{0}$ is the magnetic flux quantum, $j_{c}$ is the critical current density. Equation (1) were derived from first principles in Ref. 8, where the notations $s$ and $d^{\prime}$ were introduced as the effective coupling length (formally, $s<0$ ) and the effective magnetic thickness of the junctions, respectively. Note that the dimensionless coupling parameter $S<0$ is determined completely by the thickness of the insulating and superconducting layers in the stacked junctions. A typical experimental value for $S$ in a two junction stack may be in the interval from -0.2 to -0.7 (see, for example, Ref. 2). A schematic drawing of the two coupled long junctions is shown in Fig. 1 for both the annular geometry (a) and the overlap geometry (b).

As shown in Ref. 8, Eq. (1) lead immediately to two different fluxon modes, one with coherent (in-phase) motion of two fluxons (one in each junction), and another mode with symmetric (antiphase), but still phase-locked fluxon motion. For the coherent fluxon motion it was shown ${ }^{8}$ that the characteristic maximum fluxon velocity $\bar{c}_{+}$exceeded the velocity of light in a single junction, $\bar{c}$, i.e. (remember $S<0$ ),
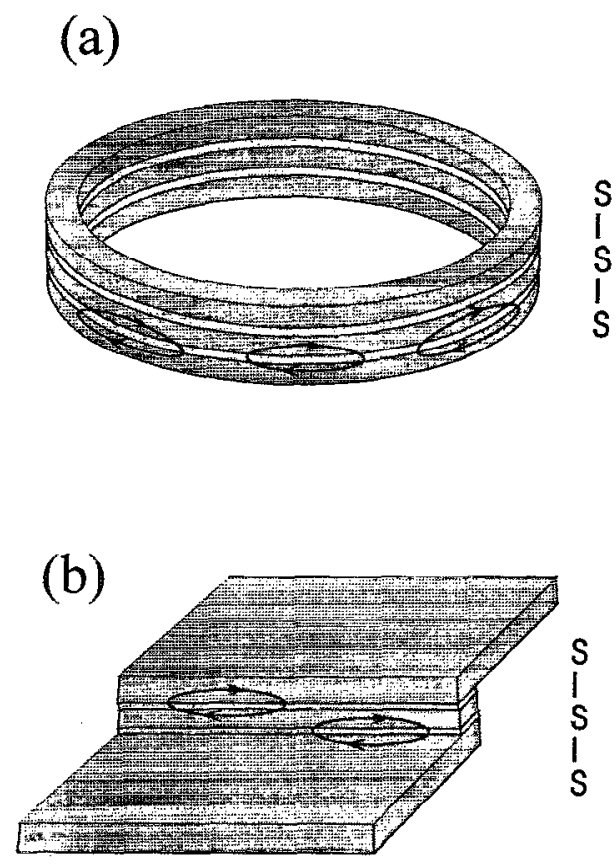

FIG. 1. Sketches of twofold long Josephson junction stacks of the annular geometry (a) and the linear overlap geometry (b).

$$
\vec{c}_{+}=\frac{\vec{c}}{\sqrt{1+S}} .
$$

It may be noticed from Ref. 8 that in the antiphase mode the characteristic velocity $\bar{c}$ - is smaller than $\bar{c}$. The splitting of the velocity of small amplitude electromagnetic waves (Swihart velocity) in a double Josephson junction stack have been predicted (in the linear approximation) many years ago by Ngai $^{16}$ but only recently observed experimentally. ${ }^{2}$ In this experiment the existence of both fluxon excitations with velocities $\bar{c}_{-}$and $\bar{c}_{+}$(in the flux flow mode) and the corresponding Fiske modes (small amplitude electromagnetic modes) were observed.

It can be shown ${ }^{17}$ from Eq. (1) that for the two junction system two Swihart modes with velocities

$$
\bar{c}_{ \pm}=\frac{\bar{c}}{\sqrt{1 \pm S}},
$$

exist. The existence of two fluxon modes can be derived also from a perturbational point of view for a general system of two weakly coupled long Josephson junctions. ${ }^{2,14}$ This result is in agreement with Eq. (3) where $S$, however, is an adjustable parameter.

In the following simulations for the two-stack long Josephson junctions the interaction of the fluxon modes and the Fiske modes with the two characteristic velocities $\bar{c}_{-}$and $\bar{c}_{+}$will play a prominent role for the observed dynamical behavior. 


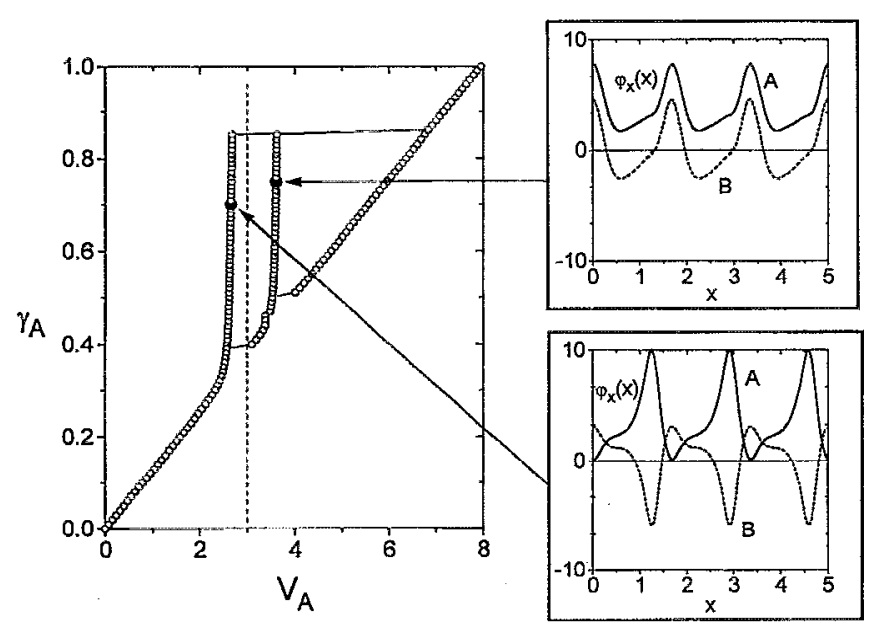

FIG. 2. Numerically calculated $I-V$ curve for a twofold stack with coupling parameter $S=-0.3$ (annular geometry) with only junction A biased $\left(\gamma_{B}=0\right), N_{A}=3$, and $N_{B}=0$. Dashed line corresponds the flux-flow step position in uncoupled single junction. Two insets show instantaneous profiles of magnetic fields $\varphi_{x}^{A}(x)$ (solid line) and $\varphi_{x}^{B}(x)$ (dashed line) in two points of the $I-V$ curve indicated by arrows.

\section{ANNULAR GEOMETRY}

In this section we present numerical simulations for the annular geometry [Fig. 1(a)]. This case correspond to Eq. (1) with periodic boundary conditions

$$
\begin{aligned}
& \left(\begin{array}{c}
\varphi_{x}^{\mathrm{A}} \\
\varphi_{x}^{\mathrm{B}}
\end{array}\right)_{x=0}=\left(\begin{array}{c}
\varphi_{x}^{\mathrm{A}} \\
\varphi_{x}^{\mathrm{B}}
\end{array}\right)_{x=L}, \\
& \left(\begin{array}{c}
\varphi^{\mathrm{A}} \\
\varphi^{\mathrm{B}}
\end{array}\right)_{x=L}=\left(\begin{array}{c}
\varphi^{\mathrm{A}} \\
\varphi^{\mathrm{B}}
\end{array}\right)_{x=0}+2 \pi\left(\begin{array}{c}
N_{A} \\
N_{B}
\end{array}\right),
\end{aligned}
$$

where $N_{A}$ and $N_{B}$ are numbers of fluxons trapped in junctions $A$ and $B$, respectively. Under the influence of bias current fluxons (which have to be trapped in the rings) perform revolutions in the junction, thus yielding a non-zero dc voltage. The advantage of the annular geometry is that there are no fluxon collisions with boundaries, and the behavior is mainly characterized by the interaction between fluxon arrays which belong to different junctions in the stack. For the sake of convenience, here we normalize the calculated voltage $V=\Delta \varphi / \Delta t$ to the single soliton step voltage $V_{0}=2 \pi \vec{c} / L$ of a conventional single-layer annular junction.

In order to identify most characteristic features in the dynamics, we investigated numerically several cases with fluxons present in one or both junctions which can be biased in various ways.

The case of $N_{A} \neq 0$ and $N_{B}=0$ is shown in Fig. 2. In spite of the fact that junction $B$ remains to be unbiased and contains no fluxons, its influence via the coupling factor $S=-0.3$ on fluxons in junction A is evident. We find two distinct relativistic branches for fluxons moving in junction A. These branches are associated with the splitting of the dispersion relation for linear waves in the structure and cor-

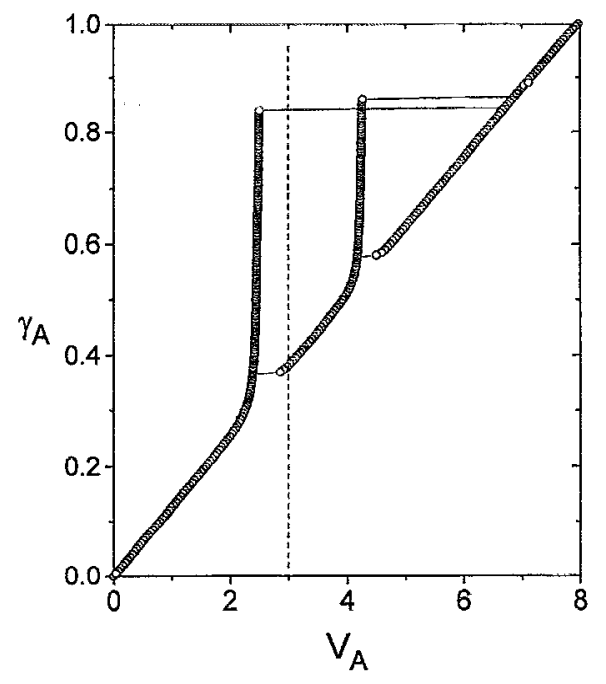

FIG. 3. $I-V$ curve of twofold stack with $S=-0.5$ (annular geometry) with junction A biased, $N_{A}=3$ and $N_{B}=0$. Dashed line corresponds the flux-flow step position in uncoupled single junction.

respond to two limiting velocities $\bar{c}_{-}$and $\bar{c}_{+}$given by Eq. (3). Dashed line in Fig. 2 shows the flux-flow step position $\left(V=N_{A}\right.$ in normalized units) in the uncoupled single junction (Swihart velocity $\bar{c}$ ). As predicted by Eq. (3), one of the velocities $\vec{c}_{-}$is below the velocity $\bar{c}$ and the other one $\bar{c}_{+}$is above it.

Two insets in Fig. 2 show a typical instantaneous profiles of magnetic fields $\varphi_{x}^{A}(x)$ (solid line) and $\varphi_{x}^{B}(x)$ (dashed line) corresponding to the modes $\bar{c}_{-}$and $\bar{c}_{+}$. Arrows indicate points in $I-V$ curve where these profiles where taken. We can sce that each fluxon moving in junction A produce a strong magnetic field perturbation, its "image," in junction B. The most distinct feature is that this image has opposite polarities for the low $\left(\bar{c}_{-}\right)$and the high $\left(\bar{c}_{+}\right)$branches. In the first case each fluxon in $\mathrm{A}$ induce a maximum in $\varphi_{x}^{B}(x)$ of the same polarity, whereas for the second case a sharp minimum is clearly observed. Note, that in both cases the spatially average $\varphi_{x}^{B}$ remains to be zero $\left(N_{B}=0\right)$.

The influence of the coupling strength on $I-V$ curve is shown in Fig. 3 for the same parameters as Fig. 2 but $S=-0.5$. In agreement with Eq. (3), increasing of the modulus of the coupling parameter produce larger splitting of the flux-flow steps in $I-V$ curve, $\bar{c}_{-}$is decreasing and $\bar{c}_{+}$is increasing.

Simulation results for $N_{A}=N_{B}=3$ are presented in Fig. 4. In this case both junctions were biased in series $\left(\gamma_{A}=\gamma_{B}\right)$ and their voltages were always found to be equal. As compared with Fig. 2, the steps in Fig. 4 appear to be high which emphasizes a strong phase locking of oscillations in two junctions. As seen from the lower inset in Fig. 4, the relativistic branch $\vec{c}_{-}$corresponds to an out-of-phase locking of fluxon arrays. We note that the antiphase arrangement of fluxon arrays in two stacked junctions is the only stable configuration in the static case $\left(\gamma_{A}=\gamma_{B}=0\right)$. Due to mutual repulsion between fluxons belonging to different junctions the static fluxon arrays are expected to be shifted by half of 


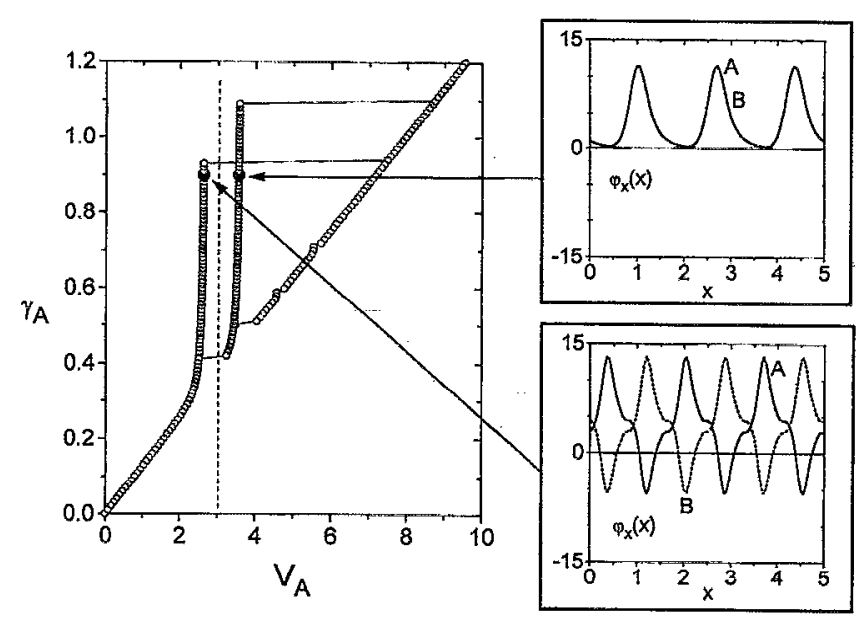

FIG. 4. The same as Fig. 2 with both junctions biased $\left(\gamma_{A}=\gamma_{B} \neq 0\right)$, $N_{A}=3$ and $N_{B}=3$. Naturally, $V_{B}=V_{A}$ is found.

their spatial period. Thus, the static out-of-phase configuration remains to be stable in dynamics for the lower relativistic branch.

For the high relativistic branch (upper inset in Fig. 4) we find a perfect in-phase locking, fluxons in $\mathrm{A}$ and $\mathrm{B}$ attract each other and the magnetic field profiles $\varphi_{x}^{A}(x)$ and $\varphi_{x}^{B}(x)$ are undistinguishable. This dynamic state appears to be very stable which is reflected in the large current range of the calculated step in Fig. 4. Our numerical result for the inphase locking is in agreement with the stability analysis by Grønbech-Jensen et al. for the bunched single-fluxon solutions in coupled junctions. ${ }^{14}$ We note also, that the in-phase (attractive) and the out-of-phase (repulsive) fluxon configurations in the stack can be understood consistently with the behavior seen from insets in Fig. 2. When the fluxon "image" in the idle junction has the same polarity as the fluxon itself one expects to have an attractive potential for a fluxon of the same polarity in the idle junction, in the opposite case the repulsion is expected.

Finally, Fig. 5 shows the simulation results for the other phase-locking case, with junctions $\mathrm{A}$ and $\mathrm{B}$ opposite biased $\left(\gamma_{A}=-\gamma_{B}\right)$ and containing fluxons of opposite polarities $\left(N_{A}=3, N_{B}=-3\right)$. Under the influence of the opposite bias currents the fluxon array in A and the array of antifluxons in $B$ move in the same direction. In contrast to Fig. 4, now the most stable dynamic state appears to be the lower relativistic branch (as can be judged from the large current range of the step). As shown in the inset, the fluxons in junction $A$ are locked in-phase with antifluxons in junction B. For the high branch $\left(\bar{c}_{\dot{+}}\right)$ (see the lower inset), as one may expect, the in-phase locking is broken and there is a phase shift between two moving lluxon arrays. Now, however, the shifted configuration of fluxon arrays is not exactly antiphase (as it was in Fig. 4). This can be related to the absence of any other stable out-of-phase configuration in this system (like the static one for the fluxon arrays of the same polarity).

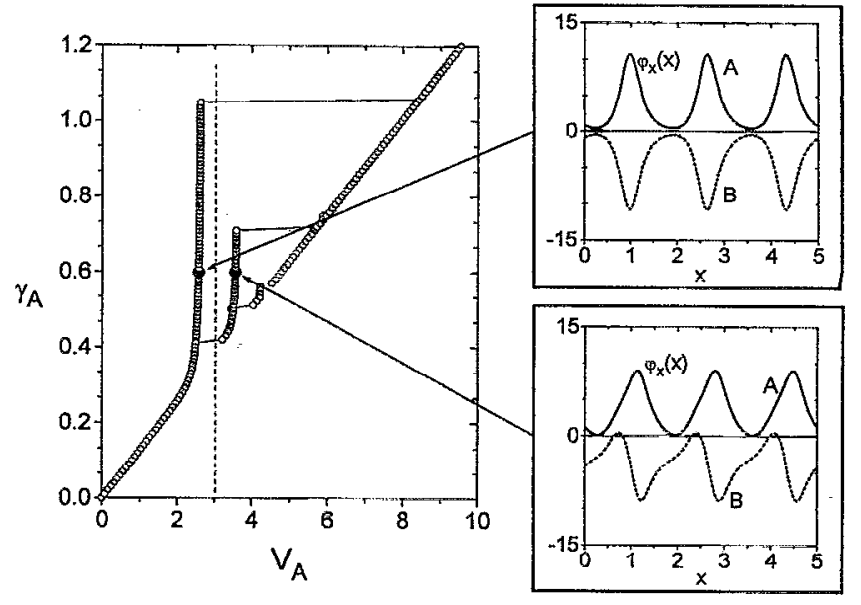

FIG. 5. The same as Fig. 2 with opposite-biased junctions $\left(\gamma_{A}=-\gamma_{B}\right.$ $\neq 0)$. Three fluxon trapped in $\mathrm{A}\left(N_{A}=3\right)$ and three antifluxons in $\mathrm{B}$ $\left(N_{B}=3\right) . V_{B}=-V_{A}$ is found.

\section{LINEAR OVERLAP GEOMETRY}

The other geometry we considered is the linear overlap geometry [Fig. 1(b)]. This geometry is interesting both from the point of view to explore the different possibilities for the dynamics but also because it is more realistic for a comparison with experiments. ${ }^{2,4}$ Before proceeding to the stacked overlap junctions we will dwell a little with the single junctions of the same type. The most prominent single dynamic features observable as singularities in the $I-V$ curve are: (i) zero-field steps (ZFS), (ii) flux-flow steps (FFS), and (iii) Fiske steps (FS).

For the zero-field steps the fluxons perform a back and forth motion due to the collisions with the boundaries; at each collision a fluxon (antifluxon) is changed into an antifluxon (fluxon). The flux-flow steps and the Fiske steps are excited by a external magnetic field that acts through the boundaries of the junction.

The FFS corresponds to the continuous injection of fluxons from one edge of the junction and their annihilation at the other boundary. The FS regime can be understood as a cavity resonance on the junction length excited by moving fluxon chain. At high enough magnetic field, such a standingwave pattern can be fairly well described by the linear mode approximation. ${ }^{18}$ For all the above phenomena the voltages of the ZFS, the FFS, and the FS are given by the Swihart velucity of the junction.

For the two-junction stack the appearance of two limiting velocities instead of the single Swihart velocity (as discussed in Sec. II) has recently been studied both analytically and numerically. ${ }^{8,14}$ It has been also observed experimentally for two stacked junctions biased in series ${ }^{2}$ and for a single biased junction in a two-junction stack. ${ }^{4}$ In those experiments the usual single-junction FFSs and FSs have been found to be replaced by more complicated $I-V$ characteristics that apparently involves two limiting velocities given by Eq. (3). For some yet not obvious reason the ZFSs have been difficult to observe experimentally. 


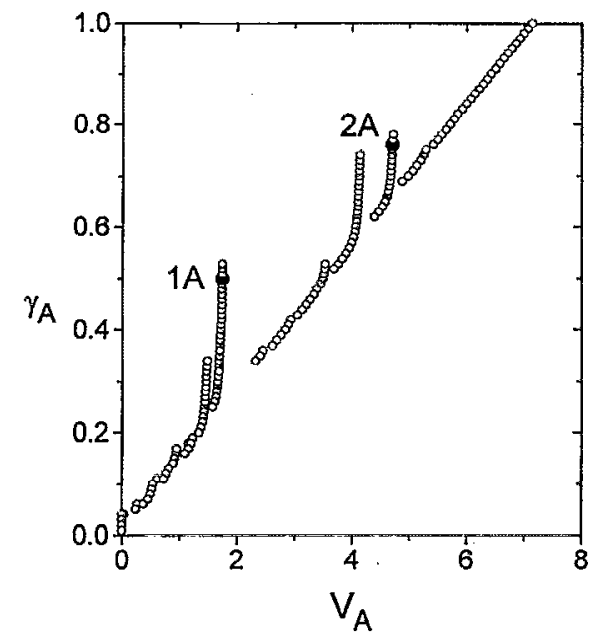

FIG. 6. Numerically calculated $I-V$ curve for a twofold stack of linear overlap geometry with only junction A biased.

In order to model the flux-flow behavior in a twojunction stack of the linear overlap geometry we have used the normalized two-junction form of Eq. (14) from Ref. 8

$$
\left(\begin{array}{c}
\varphi_{x}^{\mathrm{A}} \\
\varphi_{x}^{\mathrm{B}}
\end{array}\right)_{x=0, L}=-\eta\left(1+S+S_{e}\right)=-\eta_{\mathrm{eff}},
$$

where $\eta=H /\left(j_{c} \lambda_{J}\right)$ is the normalized external magnetic field $H$, and $S_{e}=\lambda_{L} / \sinh \left(t_{c} / \lambda_{L}\right)$. For comparison with experiments $S$ and $S_{e}$ can be calculated from the experimental parameters.

In the experiments ${ }^{2}$ the two junctions are biased in series with the same bias current. This is the geometry that is most favorable for observing phase locking of the two junctions and in addition it eliminates the difficult task of attaching leads to the center film of the structure.
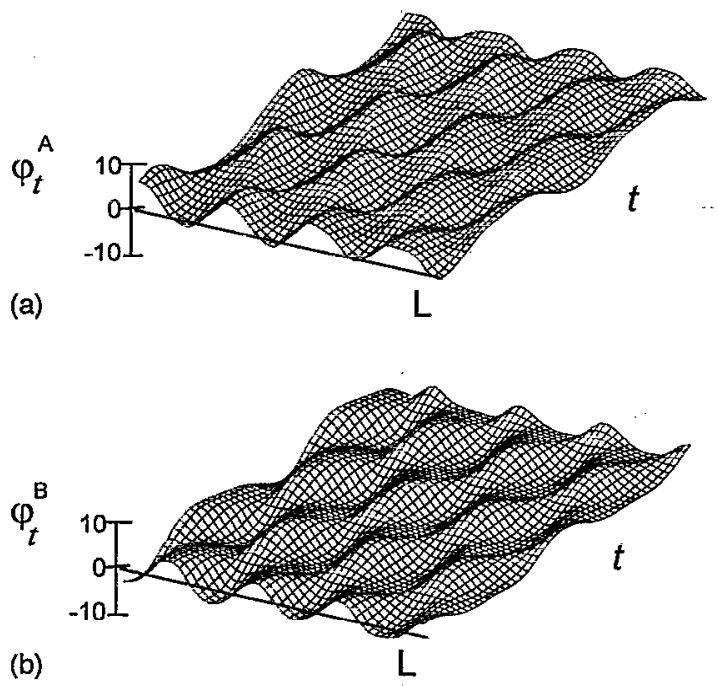

TABLE I. Summary of the numerically determined flux-flow step voltages and Fiske step voltage spacings. The theoretical ratio is 0.42 .

\begin{tabular}{ccccccc}
\hline \hline Figure & $V_{\text {FF1 }}$ & $V_{\text {FF2 }}$ & Ratio & $\Delta V_{\text {FS1 }}$ & $\Delta V_{\text {FS2 }}$ & Ratio \\
\hline 6 & 4.71 & 1.74 & 0.37 & 0.58 & 0.28 & 0.48 \\
8 & 4.14 & 1.74 & 0.42 & 0.59 & 0.24 & 0.41 \\
\hline
\end{tabular}

We have performed a series of simulations where we have changed the biasing conditions for the two junctions $\left(\gamma_{\mathrm{A}}\right.$ and $\left.\gamma_{\mathrm{B}}\right)$, the magnetic field $(\eta)$, the damping $(\alpha)$, and the coupling parameter $(S)$. As an example we show in Fig. 6 a case in which only one junction is biased $\left(\gamma_{B}=0\right)$. The figure shows the calculated normalized $I-V$ curve for junction A for fixed external magnetic field ( $\left.\eta_{\mathrm{eff}}=11.2\right)$ while the other parameters are $L=7, \alpha=0.1$, and $S=-0.7$. This situation corresponds to a stack with an electrical contact to the middle electrode, where the splitting of Fiske steps in two families has also been observed in recent experiments by Barbara et $a l^{4}$ The above parameters have been chosen to satisfy the conditions of having rather strong cavity resonances $(\alpha L<1)$ with sufficient large FS voltage spacing. As mentioned the current across junction $B$ is zero and we found that the calculated average voltage for junction $B$ with these parameters was always zero.

In Fig. 6 we observe two separated groups of steps (at $V \approx 1.8$ and $V \approx 4$ ) corresponding to two flux-flow modes with distinctly different Swihart velocities. Each flux-flow branch is split into a series of FSs with a constant voltage spacing within this branch.

In Table I we present a comparison of the numerically calculated values and theoretically predicted ones according to Eq. (3) for the two limiting velocities $\bar{c}_{-}$and $\bar{c}_{+}$. There is a good agreement between theory and simulations in the ratios of the FFS voltages and FS voltage spacings. Even better
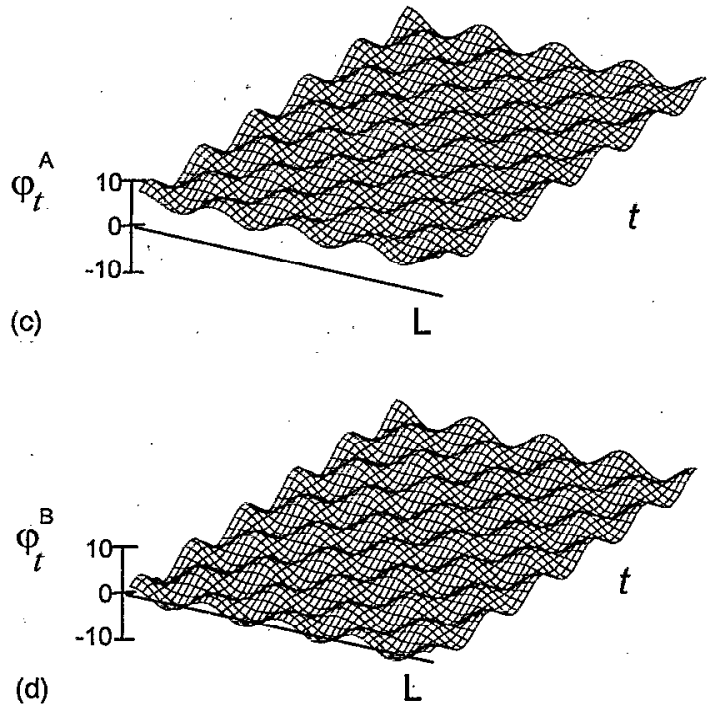

FIG. 7. Spatio-temporal voltage dynamics $\varphi_{t}(x, t)$ in different points of the calculated $I-V$ curve shown in Fig. 6: (a) junction A at point $1 \mathrm{~A}$, (b) junction B at point $1 \mathrm{~A}$, (c) junction $\mathrm{A}$ at point $2 \mathrm{~A}$, (d) junction $\mathrm{B}$ at point $2 \mathrm{~A}$. 


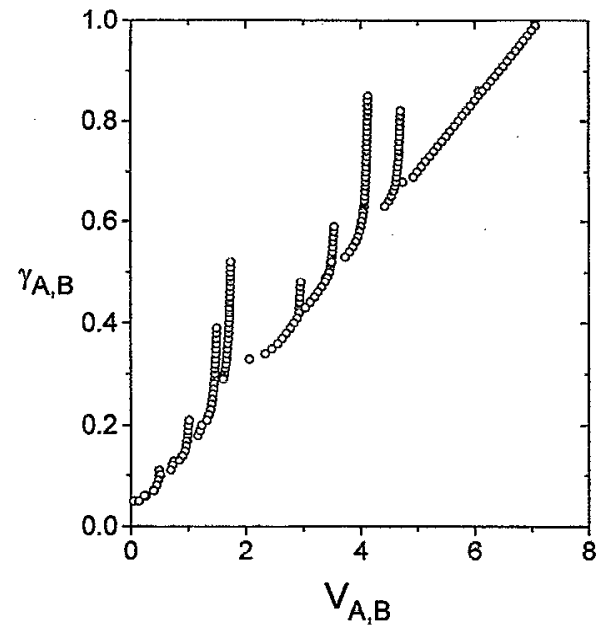

FIG. 8. Numerically calculated $I-V$ curve for a twofold stack with the same parameters as Fig. 6. Both junctions are biased.

agreement is found for the two junctions biased in series (see below).

To obtain a better understanding of the dynamics we take a closer look at the spatio-temporal voltage evolution in the stack. Figure 7 shows the normalized voltage $\varphi_{t}^{\mathrm{A}}(x, t)$ corresponding to the points marked as $1 \mathrm{~A}$ and $2 \mathrm{~A}$ in Fig. 6 [Figs. $7(\mathrm{a})$ and 7(c), respectively]. Simultaneous pictures of $\varphi_{t}^{\mathrm{B}}(x, t)$ for the unbiased junction are shown in Figs. $7(\mathrm{~b})$ and 7 (d), respectively. We observe a continuous injection of fluxons from the left edge of the stack and their motion towards the right edge. Unlike in the annular junction case, an additional spatial modulation of the voltage profiles is observed. This accounts for the standing wave pattern which is due to a cavity resonance in the Fiske mode regime. Comparing Figs. 7(a) and 7(b) we note that the two figures appear to be very similar. The standing wave pattern, however, seems to be out of phase in the two junctions. We also note that the dc voltage in the unbiased junction $B$ is zero while in junction $A$ is different from zero. Figures $7(a)$ and $7(b)$ can be superimposed and made almost identical by suitable shifts in the voltage and the time axis.

For the upper mode $\left(\bar{c}_{+}\right)$steps (point $2 \mathrm{~A}$ in Fig. 6) we find that the oscillations in the two junctions take place in phase. An example is shown in Figs. 7(c) and 7(d).

In Fig. 8 we calculated the $I-V$ curve of junction A with both junctions biased in series. The presence of the current in junction B has only a limited influence on the resonant modes in the two junctions, and in general, the appearance of the Fiske steps does not change very much. Thus the main difference between the $I-V$ curve in Fig. 8 and that of Fig. 6 is that the former displays somewhat sharper and higher Fiske steps than the latter.

A particular interest of the series biasing is due to the easy comparison between the numerical data and the experiment (see Ref. 2). In experiment two families of Fiske steps (FS1 and FS2) on two distinct flux-flow steps (FFS1 and FFS2) were observed in a very similar manner to Fig. 8. From a quantitative point of view the formula (3) seems to

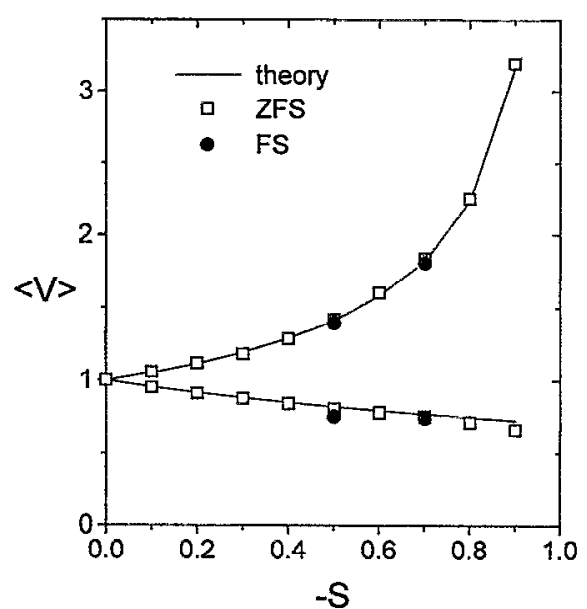

FIG. 9. Numerically determined step voltage spacings. The theoretical curve given by Eq. (3) is shown by solid line. Open squares indicate the zero-field step data, the calculated Fiske step spacings are shown by crosses.

be a good candidate to estimate the coupling parameter $S$ from the experimental current-voltage characteristics, since $\Delta V_{\mathrm{FS} 2} / \Delta V_{\mathrm{FS} 1}=\bar{c}_{+} / \bar{c}_{-}$.

In order to compare our numerical results for the fluxflow. steps and the Fiske resonances to the predictions of Eq. (3) we show in Fig. 8 the calculated normalized voltages for the upper and lower step in the case of zero-field steps. For some of the calculated Fiske steps we have also shown the normalized voltage differences. The continuous curves represent the theoretical prediction [Eq. (3)] while the symbols are the calculated results. As can be seen, a good agreement is found, in particular for zero-field steps.

\section{CONCLUSION}

In this work we reported results of numerical simulations of fluxon dynamic regimes in the system of two vertically stacked long Josephson junctions using the model by Sakai ct al. $^{8}$ We found several distinct features which have been observed in recent experiments with stacked $\mathrm{Nb} / \mathrm{Al} / \mathrm{AlO}_{x} / \mathrm{Nb}$ junctions. $^{2-4}$ In agreement with these experiments, the simulations indicate a splitting of the dispersion relation in two branches $\bar{c}_{-}$and $\bar{c}_{+}$, each corresponding to fluxons moving with different limiting (Swihart) velocity. For the mutually phase-locked fluxon arrays of the same polarity we find the stable in-phase mode at $\vec{c}_{+}$branch, and the stable out-ofphase mode for $\bar{c}$ - branch. These results support a possibility of application of the intrinsically phase-locked stacked Josephson junctions as local oscillators for millimeter and sub-millimeter wave cryoelectronic circuits.

\section{ACKNOWLEDGMENTS}

We thank P. Bodin for providing us with his original numerical code, and R. Kleiner, H. Kohlstedt, P. Müller, M. R. Samuelsen for useful discussions. 
'H. Amin, M. G. Blamire, and J. E. Evetts, IEEE Trans. Appl. Supercond. 3, 2204 (1993).

${ }^{2}$ A. V. Ustinov, H. Kohistedt, M. Cirillo, N. F. Pedersen, G. Hallmanns, and C. Heiden, Phys. Rev. B 48, 10614 (1993).

${ }^{3}$ A. V. Ustinov, H. Kohlstedt, and C. Heiden, Appl. Phys. Lett. 65, 1457 (1994).

${ }^{4}$ P. Barbara, A. V. Ustinov, and G. Costabile, Phys. Lett. A 191, 343 (1994).

${ }^{5}$ I. P. Nevirkovets, J. E. Evetts, and M. G. Blamire, Phys. Lett. A 187, 119 (1994).

${ }^{6} \mathrm{R}$. Kleiner and P. Müller, Phys. Rev. B 49, 1327 (1994).

${ }^{7}$ R. Kleiner, F. Steinmeyer, G. Kunkel, and P. Müller, Phys. Rev. Lett. 68, 2394 (1992).

${ }^{8}$ S. Sakai, P. Bodin, and N. F. Pedersen, J. Appl. Phys. 73, 2411 (1993).

${ }^{9}$ S. P. Yukun and N. C. H. Lin, IEEE Trans. Appl. Supercond. 3, 2532 (1993).
${ }^{10}$ M. B. Mineev, G. S. Mkrtchjan, and V. V. Schmidt, J. Low-Temp. Phys. 45, 497 (1981).

${ }^{11}$ A. F. Volkov, Pis'ma Zh. Exsp. Teor. Fiz. 45, 299 (1987) [JETP Lett. 45, 376 (1987)].

${ }^{12}$ Yu. S. Kivshar and B. A. Malomed, Phys. Rev. B 37, 9325 (1988).

${ }^{13}$ N. Grónbech-Jensen and J. A. Blackburn, Phys. Rev. Lett. 70, 1251 (1993); N. Gronbech-Jensen, M. R. Samuelsen, P. S. Lomdahl, and J. A. Blackburn, Phys. Rev. 42, 3976 (1990).

${ }^{14}$ N. Gronbech-Jensen, D. Cai, and M. R. Samuelsen, Phys. Rev. B 48, 16160 (1993).

${ }^{15}$ T. Holst, J. Bindslev Hansen, N. Gronbech-Jensen, and J. A. Blackburn, Phys. Rev. 42, 127 (1990).

${ }^{16}$ K. L. Ngai, Phys. Rev. 182, 555 (1969).

${ }^{17}$ S. Sakai, A. V. Ustinov, H. Kohlstedt, A. Petraglia, and N. F. Pedersen, Phys. Rev. B 50, 12905 (1994).

${ }^{18}$ B. Mayer, T. Doderer, R. P. Huebener, and A. V. Ustinov, Phys. Rev. B 44, 12463 (1991). 